\title{
On the Darboux integrability of the Painlevé II equations
}

Jaume Llibre, Claudia Valls

To cite this article: Jaume Llibre, Claudia Valls (2015) On the Darboux integrability of the Painlevé II equations, Journal of Nonlinear Mathematical Physics 22:1, 60-75, DOI: https://doi.org/10.1080/14029251.2015.996441

To link to this article: https://doi.org/10.1080/14029251.2015.996441

Published online: 04 January 2021 


\title{
On the Darboux integrability of the Painlevé II equations
}

\author{
Jaume Llibre \\ Departament de Matemàtiques, Universitat Autònoma de Barcelona \\ 08193 Bellaterra, Barcelona, Catalonia, Spain \\ jllibre@mat.uab.cat \\ Claudia Valls \\ Departamento de Matemática, Instituto Superior Técnico \\ 1049-001 Lisboa, Portugal \\ cvalls@math.ist.utl.pt
}

Received 10 July 2014

Accepted 26 September 2014

In this paper we prove the non-existence of Darboux first integrals for the Painlevé II equations

$$
\dot{x}=y-\frac{z}{2}-x^{2}, \quad \dot{y}=\alpha+\frac{1}{2}+2 x y, \quad \dot{z}=1
$$

for all values of $\alpha \in \mathbb{C} \backslash\left\{\alpha_{n}: n=2,4, \ldots\right\}$. These $\alpha_{n}$ are real and larger than $-1 / 2$.

Keywords: Painlevé transcendents; Hamiltonian systems; Darboux integrability.

\section{Introduction and statement of the main results}

The Painlevé equations are Hamiltonian systems that depend on parameters and whose solutions give rise to the so-called Painlevé transcendents. The Painlevé transcendents are solutions to certain nonlinear second-order ordinary differential equations in the complex plane whose only movable singularities are ordinary poles and which cannot be integrated in terms of other known functions or transcendents.

In this paper we study the Darboux integrability of the Painlevé II equations which can be written in the form (see [2-5] for details):

$$
\dot{x}=y-\frac{z}{2}-x^{2}, \quad \dot{y}=\alpha+\frac{1}{2}+2 x y, \quad \dot{z}=1, \quad \dot{w}=\frac{y}{2}
$$

with the Hamiltonian

$$
H=\frac{1}{2} y^{2}-\left(x^{2}+\frac{z}{2}\right) y-\left(\alpha+\frac{1}{2}\right) x+w .
$$

Here the parameter $\alpha$ is complex. Using $H$ we can eliminate the variable $w$ making

$$
w=H-\frac{1}{2} y^{2}+\left(x^{2}+\frac{z}{2}\right) y+\left(\alpha+\frac{1}{2}\right) x,
$$

and study the integrability of the system

$$
\dot{x}=y-\frac{z}{2}-x^{2}, \quad \dot{y}=\alpha+\frac{1}{2}+2 x y, \quad \dot{z}=1 .
$$


We note that with $z(t)=t$ it is the variable $x(t)$ of system (1.1) which satisfies the second Painleve equation in its usual form, and gives this second order equation.

Systems (1.1) were previously studied by Morales in [4] (see Corollary 1) where the author proved:

Theorem 1.1 (Morales' result). For $\alpha \in \mathbb{Z}$ system (1.1) is not integrable by means of rational first integrals.

We define for each positive even integer $n \geq 2$ the value $\alpha_{n}$ as the zero of

$$
\frac{\left(\alpha+\frac{1}{2}\right) n \prod_{j=0}^{\frac{n}{2}-1}(n-2 j)}{\prod_{i=1}^{\frac{n}{2}}(4 i)}-D_{n / 2}=0,
$$

where $D_{n / 2}$ are positive real numbers defined in the proof of Proposition 3.1. We improve Morales' result as follows.

Theorem 1.2. For all $\alpha \in \mathbb{C} \backslash\left\{\alpha_{n}: n=2,4, \ldots\right\}$ system (1.1) is not integrable by means of a Darboux first integral.

Theorem 1.2 is proved in section 5 .

We note that a rational first integral is a very particular case of a first integral of Darboux type (see (2.4) for its definition).

Theorem 1.3. The following holds for system (1.1).

(a) If $\alpha=-1 / 2$, the unique irreducible Darboux polynomial with non-zero cofactor is $y$. Its cofactor is $2 x$.

(b) If $\alpha \in \mathbb{C} \backslash\left\{\alpha_{n}: n=2,4, \ldots\right\}$ and $\alpha \neq-1 / 2$, system (1.1) has no irreducible Darboux polynomials with non-zero cofactor.

(c) Assume $\alpha \in \mathbb{C} \backslash\left\{\alpha_{n}: n=2,4, \ldots\right\}$.

(c.1) System (1.1) has no polynomial first integrals.

(c.2) The exponential factors $e^{z}, e^{z^{2} / 2}$ and $e^{2 y x^{2}+(2 \alpha+1) x-y^{2}+z y}$ are the unique ones for system (1.1) having cofactors $1, z$ and $y$, respectively.

Theorem 1.3 is proved in section 4 .

In sections 2 and 3 we present basic definitions, notations, some preliminary and auxiliary results.

\section{Preliminary results}

Consider a polynomial differential system

$$
\dot{x}=P_{1}(x, y, z), \quad \dot{y}=P_{2}(x, y, z), \quad \dot{z}=P_{3}(x, y, z),
$$

where $P_{i}=P_{i}(x, y, z) \in \mathbb{R}[x, y, z]$ are polynomials of degree at most two and its associated vector field is

$$
\mathbf{X}=P_{1} \frac{\partial}{\partial x}+P_{2} \frac{\partial}{\partial y}+P_{3} \frac{\partial}{\partial z} .
$$

Let $U \subset \mathbb{R}^{3}$ be an open subset. We say that the non-constant function $H: U \rightarrow \mathbb{R}$ is a first integral of the polynomial vector field (2.2) on $U$ associated to system (2.1), if $H(x(t), y(t), z(t))=$ 
constant for all values of $t$ for which the solution $(x(t), y(t), z(t))$ of $\mathbf{X}$ is defined on $U$. Clearly $H$ is a first integral of $\mathbf{X}$ on $U$ if and only if $\mathbf{X} H=0$ on $U$. When $H$ is a polynomial we say that $H$ is a polynomial first integral.

Let $h=h(x, y, z) \in \mathbb{C}[x, y, z]$ be a nonconstant polynomial. We say that $h=0$ is an invariant algebraic surface of the vector field $\mathbf{X}$ if it satisfies $\mathbf{X} h=K h$ for some polynomial $K=K(x, y, z) \in$ $\mathbb{C}[x, y, z]$, called the cofactor of $h=0$. Note that $K$ has degree at most 1 . The polynomial $h$ is called a Darboux polynomial, and we also say that $K$ is the cofactor of the Darboux polynomial $h$. We note that a Darboux polynomial with zero cofactor is a polynomial first integral.

Let $g, h \in \mathbb{C}[x, y, z]$ be coprime. We say that a nonconstant function $E=e^{h / g}$ is an exponential factor of the vector field $X$ if it satisfies $\mathbf{X} E=L E$ for some polynomial $L=L(x, y, z) \in \mathbb{C}[x, y, z]$, called the cofactor of $E$ and having degree at most 1. Note that this relation is equivalent to

$$
P_{1} \frac{\partial(g / h)}{\partial x}+P_{2} \frac{\partial(g / h)}{\partial y}+P_{3} \frac{\partial(g / h)}{\partial z}=L .
$$

For a geometric and algebraic meaning of the exponential factors see [1].

A first integral $G$ of system (2.1) is called of Darboux type if it is of the form

$$
G=f_{1}^{\lambda_{1}} \cdots f_{p}^{\lambda_{p}} E_{1}^{\mu_{1}} \cdots E_{q}^{\mu_{q}}
$$

where $f_{1}, \ldots, f_{p}$ are Darboux polynomials, $E_{1}, \ldots, E_{q}$ are exponential factors and $\lambda_{j}, \mu_{k} \in \mathbb{C}$ for $j=1, \ldots, p, k=1, \ldots, q$.

For a proof of the next proposition see [1].

Proposition 2.1. The following statements hold.

(a) If $E=e^{g / h}$ is an exponential factor for the polynomial differential system (2.1) and $h$ is not a constant polynomial, then $h=0$ is an invariant algebraic surface.

(b) Eventually $e^{g}$ can be an exponential factor, coming from the multiplicity of the infinite invariant plane.

\section{Auxiliary result}

The next result plays a key role in the proof of our two theorems.

Proposition 3.1. Assume $\alpha \in \mathbb{C} \backslash\left\{\alpha_{n}: n=2,4, \ldots,\right\}$. Then system (1.1) has no first integrals which are polynomials in the variable $x$, analytic in the variable $z$ and have a decomposition in powers in the variable $y$.

Proof. Let $g=g(x, y, z)$ be a first integral of system (1.1) which is polynomial in the variable $x$, analytic in the variable $z$ and have a decomposition in powers of the variable $y$. Then $g$ is not a constant and satisfies

$$
\left(y-\frac{z}{2}-x^{2}\right) \frac{\partial g}{\partial x}+\left(\alpha+\frac{1}{2}+2 x y\right) \frac{\partial g}{\partial y}+\frac{\partial g}{\partial z}=0 .
$$

Since $g$ is a polynomial in the variable $x$, we rewrite it as

$$
g(x, y, z)=\sum_{j=0}^{n} g_{j}(y, z) x^{j}
$$


where each $g_{j}$ are functions in the variables $y, z$. We have that equation (3.1) becomes

$$
\begin{aligned}
& -\sum_{j=0}^{n} j g_{j}(y, z) x^{j+1}+2 \sum_{j=0}^{n} y \frac{\partial g_{j}(y, z)}{\partial y} x^{j+1}+\sum_{j=0}^{n} \frac{\partial g_{j}(y, z)}{\partial z} x^{j} \\
& +\sum_{j=0}^{n}\left(\alpha+\frac{1}{2}\right) \frac{\partial g_{j}(y, z)}{\partial y} x^{j}+\sum_{j=0}^{n} j\left(y-\frac{z}{2}\right) g_{j}(y, z) x^{j-1}=0 .
\end{aligned}
$$

Now we will show by induction that for $l=0, \ldots,[n / 2]$,

$$
g_{n-2 l}(y, z)=\frac{(-1)^{l}}{\prod_{i=1}^{l}(4 i)} K(z) y^{\frac{n}{2}+l} \prod_{j=0}^{l-1}(n-2 j)+\text { l.o.t. }(y),
$$

where 1.o.t. $(y)$ means terms with lower power in the variable $y,[\cdot]$ denotes the integer part function of a real number, $K(z)$ is a function in the variable $z$ and we have taken the convention that $\prod_{i=1}^{0} \cdot=1$ and $\prod_{j=0}^{-1} \cdot=1$. Furthermore, we will also show by induction that for $l=0, \ldots,[(n-1) / 2]$,

$$
g_{n-2 l-1}(y, z)=(-1)^{l+1} C_{l} K^{\prime}(z) y^{\frac{n}{2}+l}+\text { l.o.t. }(y),
$$

where $C_{l}$ are positive constants for each $l=0, \ldots,[(n-1) / 2]$.

Computing the terms of degree $n+1$ in $x$ in (3.3) we get

$$
-n g_{n}(y, z)+2 y \frac{\partial g_{n}(y, z)}{\partial y}=0 \quad \text { that is } \quad g_{n}(y, z)=K(z) y^{n / 2}
$$

where $K(z)$ is a function in the variable $z$. This proves (3.4) for $l=0$. Now computing the terms of degree $n$ in $x$ in (3.3) we obtain

$$
-(n-1) g_{n-1}(y, z)+2 y \frac{\partial g_{n-1}(y, z)}{\partial y}+K^{\prime}(z) y^{n / 2}+\left(\alpha+\frac{1}{2}\right) \frac{n}{2} K(z) y^{n / 2-1}=0 .
$$

Solving this linear differential equation we obtain

$$
\begin{aligned}
g_{n-1}(y, z) & =K_{1}(z) y^{(n-1) / 2}-K^{\prime}(z) y^{n / 2}+\frac{n(1+2 \alpha)}{4} K(z) y^{n / 2-1} \\
& =-K^{\prime}(z) y^{n / 2}+\log (y)
\end{aligned}
$$

where $K_{1}$ is some function in the variable $z$. This proves (3.5) for $l=0$ with $C_{0}=1$.

Now we proceed by induction. We assume that (3.4) and (3.5) are true for $l=0, \ldots, \ell$ and we will show it for $\ell+1$. 
Computing the terms of degree $n-2 \ell-1$ in (3.3) we get that

$$
\begin{aligned}
0 & =-(n-2 \ell-2) g_{n-2 \ell-2}+2 y \frac{\partial g_{n-2 \ell-2}}{\partial y}+(-1)^{\ell+1} C_{\ell} K^{\prime \prime}(z) y^{\frac{n}{2}+\ell}+1 . o . t(y) \\
& +\left(y-\frac{z}{2}\right)(n-2 \ell)\left(\frac{(-1)^{\ell} K(z) y^{\frac{n}{2}+\ell} \prod_{j=0}^{\ell-1}(n-2 j)}{\prod_{i=1}^{\ell}(4 i)}+1 . o . t(y)\right) \\
& +\left(\alpha+\frac{1}{2}\right)\left(\left(\frac{n}{2}+\ell\right)(-1)^{\ell+1} C_{l} K^{\prime}(z) y^{\frac{n}{2}+\ell-1}+1 . o . t(y)\right) \\
& =-(n-2 \ell-2) g_{n-2 \ell-2}+2 y \frac{\partial g_{n-2 \ell-2}}{\partial y}+(n-2 \ell) \frac{(-1)^{\ell} K(z) y^{\frac{n}{2}+\ell+1} \prod_{j=0}^{\ell-1}(n-2 j)}{\prod_{i=1}^{\ell}(4 i)} \\
& + \text { l.o.t }(y) \\
& =-(n-2 \ell-2) g_{n-2 \ell-2}+2 y \frac{\partial g_{n-2 \ell-2}}{\partial y}+\frac{(-1)^{\ell} K(z) y^{\frac{n}{2}+\ell+1} \prod_{j=0}^{\ell}(n-2 j)}{\prod_{i=1}^{\ell}(4 i)}+\text { l.o.t }(y) .
\end{aligned}
$$

Then solving this linear differential equation we conclude that

$$
\begin{aligned}
g_{n-2 \ell-2} & =\frac{-(-1)^{\ell} K(z)}{4(\ell+1) \prod_{i=1}^{\ell}(4 i)} y^{\frac{n}{2}+\ell+1} \prod_{j=0}^{\ell}(n-2 j)+\text { 1.o.t. }(y) \\
& =\frac{(-1)^{\ell+1} K(z)}{\prod_{i=1}^{\ell+1}(4 i)} y^{\frac{n}{2}+\ell+1} \prod_{j=0}^{\ell}(n-2 j)+\text { l.o.t. }(y),
\end{aligned}
$$

which proves (3.4) for $l=\ell+1$.

Now computing the terms of degree $n-2 \ell-2$ in (3.3) we get that

$$
\begin{aligned}
0 & =-(n-2 \ell-3) g_{n-2 \ell-3}+2 y \frac{\partial g_{n-2 \ell-3}}{\partial y}+\frac{(-1)^{\ell+1}}{\prod_{i=1}^{\ell+1}(4 i)} K^{\prime}(z) y^{\frac{n}{2}+\ell+1} \prod_{j=0}^{\ell}(n-2 j)+\text { l.o.t }(y) \\
& +\left(y-\frac{z}{2}\right)(n-2 \ell-1)\left((-1)^{\ell+1} C_{\ell} K^{\prime}(z) y^{\frac{n}{2}+\ell}+1 . \text { o.t }(y)\right) \\
& +\left(\alpha+\frac{1}{2}\right)\left(\left(\frac{n}{2}+\ell+1\right) \frac{(-1)^{\ell+1}}{\prod_{i=1}^{\ell+1}(4 i)} K(z) y^{\frac{n}{2}+\ell} \prod_{j=0}^{\ell}(n-2 j)+1 . o . t(y)\right) \\
& =-(n-2 \ell-3) g_{n-2 \ell-3}+2 y \frac{\partial g_{n-2 \ell-3}}{\partial y}+(-1)^{\ell+1} K_{\ell} K^{\prime}(z) y^{\frac{n}{2}+\ell+1}+\operatorname{log.t}(y)
\end{aligned}
$$

where

$$
K_{\ell}=\frac{\prod_{j=0}^{\ell}(n-2 j)}{\prod_{i=1}^{\ell+1}(4 i)}+(n-2 \ell-1) C_{\ell}>0 .
$$

Then solving this linear differential equation we obtain that

$$
g_{n-2 \ell-3}=(-1)^{\ell+2} C_{\ell+1} K^{\prime}(z) y^{n / 2+\ell+1}+\text { l.o.t. }(y),
$$

where $C_{\ell+1}=K_{\ell} /(4 \ell+5)$. This proves (3.5) for $l=\ell+1$. Hence (3.4) and (3.5) are proved. Now computing the terms of degree 0 in $x$ in (3.3) we get

$$
\frac{\partial g_{0}}{\partial z}+\left(\alpha+\frac{1}{2}\right) \frac{\partial g_{0}}{\partial y}+\left(y-\frac{z}{2}\right) g_{1}=0
$$


Now we distinguish two cases.

Case 1: $n$ is odd. Using (3.4) and (3.5) with $\ell=(n-1) / 2$ we get that (3.8) becomes

$$
(-1)^{\frac{n-1}{2}} \frac{\prod_{j=0}^{\frac{n-3}{2}}(n-2 j)}{\prod_{i=1}^{\frac{n-1}{2}}(4 i)} K(z) y^{\frac{2 n+1}{2}}+\text { l.o.t }(y)=0,
$$

which yields $K(z)=0$. Hence $g_{n}=0$ for $n \geq 1$ and then, from (3.2), $g=g(y, z)$. This implies, from (3.1), that

$$
\left(\alpha+\frac{1}{2}+2 x y\right) \frac{\partial g}{\partial y}+\frac{\partial g}{\partial z}=0
$$

Since $g$ does not depend on $x$, this identity implies that $\frac{\partial g}{\partial y}=\frac{\partial g}{\partial z}=0$. Therefore $g=$ constant, a contradiction with the fact that $g$ is a first integral.

Case 2: $n$ is even. In this case using (3.4) with $\ell=n / 2$ and (3.5) with $\ell=(n-2) / 2$ we get that (3.8) becomes

$$
\begin{aligned}
& (-1)^{\frac{n}{2}} \frac{\prod_{j=0}^{\frac{n-2}{2}}(n-2 j)}{\prod_{i=1}^{\frac{n}{2}}(4 i)} K^{\prime}(z) y^{n}+1.0 . t(y)+(-1)^{\frac{n-2}{2}+1} C_{\frac{n-2}{2}} K^{\prime}(z) y^{\frac{n}{2}+\frac{n-2}{2}+1}+\text { l.o.t }(y) \\
& =(-1)^{\frac{n}{2}} K^{\prime}(z) y^{n}\left(\frac{\prod_{j=0}^{\frac{n-2}{2}}(n-2 j)}{\prod_{i=1}^{\frac{n}{2}}(4 i)}+C_{\frac{n-2}{2}}\right)+\text { l.o.t }(y)=0 .
\end{aligned}
$$

Since

$$
\frac{\prod_{j=0}^{\frac{n-2}{2}}(n-2 j)}{\prod_{i=1}^{\frac{n}{2}}(4 i)}+C_{\frac{n-2}{2}}>0
$$

we get that $K^{\prime}(z)=0$ that is $K(z)=\gamma_{n}$, where $\gamma_{n} \in \mathbb{C}$.

Now we will show by induction with respect to $l$ that for $n \geq 2$ and $l=1,2, \ldots,[n / 2]$,

$$
g_{n-2 l}(y, z)=\frac{(-1)^{l}}{\prod_{i=1}^{l}(4 i)} \gamma_{n} y^{\frac{n}{2}+l} \prod_{j=0}^{l-1}(n-2 j)+(-1)^{l+1} \gamma_{n} D_{l} z y^{\frac{n}{2}+l-1}+\text { 1.o.t. }(y),
$$

where $D_{l}$ are positive constants. Note that (3.9) provides an additional term in (3.4). Furthermore, for $l=0,1, \ldots,[(n-1) / 2]$,

$$
g_{n-2 l-1}(y, z)=(-1)^{l} E_{l}(z) y^{\frac{n-1}{2}+l}+\text { l.o.t. }(y),
$$

where $E_{l}(z)$ are functions in the variable $z$ for each $l=0,1, \ldots,[(n-1) / 2]$. Note that equation (3.10) improves (3.5), because we know that the apparent dominant term in (3.5) is zero. 
We proved in (3.6) that

$$
g_{n}(y, z)=\gamma_{n} y^{n / 2} .
$$

Now from (3.7) we get

$$
-(n-1) g_{n-1}(y, z)+2 y \frac{\partial g_{n-1}(y, z)}{\partial y}\left(\alpha+\frac{1}{2}\right) \frac{n}{2} \gamma_{n} y^{n / 2-1}=0 .
$$

Solving this linear equation we obtain

$$
\begin{aligned}
g_{n-1}(y, z) & =K_{1}(z) y^{(n-1) / 2}+\frac{n(1+2 \alpha)}{4} \gamma_{n} y^{n / 2-1} \\
& =K_{1}(z) y^{(n-1) / 2}+\log (y)
\end{aligned}
$$

where $K_{1}$ is some function in the variable $z$. This proves (3.10) for $l=0$ and with $E_{1}(z)=K_{1}(z)$.

Now computing the terms of degree $n-1$ in $x$ in (3.3) we obtain

$$
-(n-2) g_{n-2}(y, z)+2 y \frac{\partial g_{n-2}(y, z)}{\partial y}+n \gamma_{n} y^{\frac{n}{2}+1}-\frac{n}{2} z \gamma_{n} y^{\frac{n}{2}}+\text { l.o.t }(y)=0 .
$$

Solving this linear equation we obtain

$$
g_{n-2}(y, z)=-\frac{n}{4} \gamma_{n} y^{\frac{n}{2}+1}+D_{1} z \gamma_{n} y^{\frac{n}{2}}+\text { l.o.t }(y)
$$

where $D_{1}=n / 4>0$. This proves (3.9) for $l=1$.

Now we shall use the induction hypothesis. We assume that (3.9) is true for $l=1,2, \ldots, \ell$ and (3.10) is true for $l=0,1, \ldots, \ell$ and we will show them for $\ell+1$.

Computing the terms of degree $n-2 \ell-1$ in $x$ of (3.3) we get that

$$
\begin{aligned}
0 & =-(n-2 \ell-2) g_{n-2 \ell-2}+2 y \frac{\partial g_{n-2 \ell-2}}{\partial y}+(-1)^{\ell} E_{\ell}^{\prime}(z) y^{\frac{n-1}{2}+\ell}+1 . o . t(y) \\
& +(y-z / 2)(n-2 \ell)\left(\frac{(-1)^{\ell} \gamma_{n} y^{\frac{n}{2}+\ell} \prod_{j=0}^{\ell-1}(n-2 j)}{\prod_{i=1}^{\ell}(4 i)}+(-1)^{\ell+1} D_{l} \gamma_{n} z y^{\frac{n}{2}+\ell-1}+\text { l.o.t }(y)\right) \\
& =-(n-2 \ell-2) g_{n-2 \ell-2}+2 y \frac{\partial g_{n-2 \ell-2}}{\partial y}+\frac{(-1)^{\ell} \gamma_{n} y^{\frac{n}{2}+\ell+1} \prod_{j=0}^{\ell}(n-2 j)}{\prod_{i=1}^{\ell}(4 i)} \\
& +(-1)^{\ell+1} \gamma_{n} F_{\ell+1} z y^{\frac{n}{2}+\ell}+1 . \operatorname{o.t}(y),
\end{aligned}
$$

where

$$
F_{\ell+1}=(n-2 \ell)\left(\frac{\prod_{j=0}^{\ell-1}(n-2 j)}{2 \prod_{i=1}^{\ell}(4 i)}+D_{\ell}\right)>0 .
$$

Then solving this linear equation we conclude that

$$
g_{n-2 \ell-2}=\frac{(-1)^{\ell+1} \gamma_{n} y^{n / 2+\ell+1}}{\prod_{i=1}^{\ell+1}(4 i)} \prod_{j=0}^{\ell}(n-2 j)+(-1)^{\ell+2} D_{\ell+1} \gamma_{n} z y^{n / 2+\ell}+\text { l.o.t. }(y),
$$

where $D_{\ell+1}=F_{\ell+1} /(2(2 \ell+1))>0$. Hence equation (3.9) is proved for $l=\ell+1$. 
Now computing the terms of degree $n-2 \ell-2$ in $x$ of (3.3) we obtain

$$
\begin{aligned}
0 & =-(n-2 \ell-3) g_{n-2 \ell-3}+2 y \frac{\partial g_{n-\ell-3}}{\partial y}+(-1)^{\ell} D_{\ell+1} \gamma_{n} y^{\frac{n}{2}+\ell}+1 . \text { o.t }(y) \\
& +(n-2 \ell-1)(-1)^{\ell} E_{\ell}(z) y^{\frac{n+1}{2}+\ell}+1.0 . t(y) \\
& =-(n-2 \ell-3) g_{n-2 \ell-3}+2 y \frac{\partial g_{n-\ell-3}}{\partial y}+(n-2 \ell-1)(-1)^{\ell} E_{\ell}(z) y^{\frac{n+1}{2}+\ell}+1 . o . t(y) .
\end{aligned}
$$

Then solving this linear equation we conclude that

$$
g_{n-2 \ell-3}=(-1)^{\ell+1} E_{\ell+1}(z) y^{\frac{n+1}{2}+\ell}+\text { 1.o.t. }(y),
$$

where $E_{\ell+1}(z)=(n-2 \ell-1) E_{\ell}(z) /(4(\ell+1))$. This proves (3.10) for $l=\ell+1$. In short, the expressions of (3.9) and (3.10) are proved.

Now using (3.9) with $\ell=n / 2$ and (3.10) with $\ell=(n-2) / 2$, we get that (3.8) becomes

$$
\begin{aligned}
& (-1)^{\frac{n}{2}+1} \gamma_{n} D_{n / 2} y^{n-1}+\frac{\left(\alpha+\frac{1}{2}\right)(-1)^{\frac{n}{2}} \gamma_{n} n y^{n-1} \prod_{j=0}^{\frac{n}{2}-1}(n-2 j)}{\prod_{i=1}^{\frac{n}{2}}(4 i)} \\
& \quad+(-1)^{\frac{n-2}{2}} E_{(n-2) / 2}(z) y^{\frac{2 n-1}{2}}+\operatorname{logt}(y) \\
& =(-1)^{\frac{n-2}{2}} E_{(n-2) / 2}(z) y^{\frac{2 n-1}{2}}+(-1)^{\frac{n}{2}} \gamma_{n} y^{n-1}\left(-D_{n / 2}+\frac{\left(\alpha+\frac{1}{2}\right) n \prod_{j=0}^{\frac{n}{2}-1}(n-2 j)}{\prod_{i=1}^{\frac{n}{2}}(4 i)}\right) \\
& \quad+\text { l.o.t }(y)=0 .
\end{aligned}
$$

Since $D_{n / 2}>0$ and $\alpha \in \mathbb{C} \backslash\left\{\alpha_{n}: n=2,4, \ldots\right\}$, we get that

$$
-D_{n / 2}+\frac{\left(\alpha+\frac{1}{2}\right) n \prod_{j=0}^{\frac{n}{2}-1}(n-2 j)}{\prod_{i=1}^{\frac{n}{2}}(4 i)} \neq 0 .
$$

Therefore from (3.12) we obtain $\gamma_{n}=0$ for any $n \geq 2$ even. Thus, from (3.11) we obtain that $g_{n}=0$ for all $n \geq 2$ even. Since $g_{n}=0$ for all $n \geq 1$ from (3.2) yields $g=g_{0}(y, z)$. It follows from (3.1) that

$$
\left(\alpha+\frac{1}{2}+2 x y\right) \frac{\partial g_{0}}{\partial y}+\frac{\partial g_{0}}{\partial z}=0
$$

which yields $\frac{\partial g_{0}}{\partial y}=\frac{\partial g_{0}}{\partial z}=0$. Then $g=$ const a contradiction with the fact that $g$ is a first integral.

\section{Proof of Theorem 1.3}

We will separate the proof of Theorem 1.3 into different propositions.

Corollary 4.1. Assume $\alpha \in \mathbb{C} \backslash\left\{\alpha_{n}: n=2,4, \ldots\right\}$. Then system (1.1) has no polynomial first integrals.

Proof. It follows directly from Proposition 3.1. 
Proposition 4.1. Assume $\alpha=-1 / 2$. Then the unique irreducible Darboux polynomial with nonzero cofactor of system (1.1) is $y$. Its cofactor is $2 x$.

Proof. It follows by direct computations that the unique irreducible Darboux polynomial of degree one of system (1.1) with non-zero cofactor is $y$. Its cofactor is $2 x$. Now we shall prove that it is the only irreducible Darboux polynomial.

Assume that $f$ is an irreducible Darboux polynomial of system (1.1) with non-zero cofactor of degree $n \geq 2$. Then it satisfies

$$
\left(y-\frac{z}{2}-x^{2}\right) \frac{\partial f}{\partial x}+2 x y \frac{\partial f}{\partial y}+\frac{\partial f}{\partial z}=\left(\alpha_{0}+\alpha_{1} x+\alpha_{2} y+\alpha_{3} z\right) f
$$

where $\left(\alpha_{0}, \alpha_{1}, \alpha_{2}, \alpha_{3}\right) \in \mathbb{C}^{4} \backslash\{(0,0,0,0)\}$. First we shall prove that $\alpha_{2}=\alpha_{3}=0$. We assume that $\alpha_{2} \neq 0$ or $\alpha_{3} \neq 0$ and we will arrive to a contradiction. We write $f$ in sum of its homogeneous parts as follows:

$$
f=\sum_{j=0}^{n} f_{j}(x, y, z)
$$

where each $f_{j}$ is a homogenous polynomial of degree $j$. Without loss of generality we can assume that $f_{n} \neq 0$. Computing the terms of degree $n+1$ in (4.1) we obtain

$$
-x^{2} \frac{\partial f_{n}}{\partial x}+2 x y \frac{\partial f_{n}}{\partial y}=\left(\alpha_{1} x+\alpha_{2} y+\alpha_{3} z\right) f_{n}
$$

So we have that $f_{n}$ must be divisible by $x$. We write $f_{n}$ as $f_{n}=x^{l} g_{l}$ where $1 \leq l \leq n$ and $g_{l}$ is a homogenous polynomial of degree $n-l$ which is not divisible by $x$ and satisfies, after simplifying by $x^{l}$,

$$
-x^{2} \frac{\partial g_{l}}{\partial x}+2 x y \frac{\partial g_{l}}{\partial y}=\left(\left(\alpha_{1}+l\right) x+\alpha_{2} y+\alpha_{3} z\right) g_{l} .
$$

If $l=n$ then $g_{n}$ is a constant, and from (4.4) we get $\left(\left(\alpha_{1}+x\right) x+\alpha_{2} y+\alpha_{3} z\right) g_{n}=0$. Since $\alpha_{2}$ or $\alpha_{3}$ is non-zero, we obtain $g_{n}=0$, and consequently $f_{n}=0$, a contradiction. So we can assume $l<n$. Again by (4.4) we have that $g_{l}$ must be divisible by $x$, which is not possible. Hence, $\alpha_{2}=\alpha_{3}=0$.

In short equation (4.1) can be written in the form

$$
\frac{d f}{d t}(x, y, z)=\left(\alpha_{0}+\alpha_{1} x\right) f(x, y, z),
$$

where the derivative is evaluated along a solution of system (1.1). We write

$$
f(x, y, z)=g(x, y, z) e^{\alpha_{0} z} y^{\alpha_{1} / 2} .
$$

Then $g$ is a polynomial in the variable $x$ and satisfies

$$
\begin{aligned}
\frac{d f}{d t}(x, y, z) & =\frac{d g}{d t}(x, y, z) e^{\alpha_{0} z} y^{\alpha_{1} / 2}+g(x, y, z)\left(\alpha_{0}+\alpha_{1} x\right) e^{\alpha_{0} z} y^{\alpha_{1} / 2} \\
& =\left(\frac{d g}{d t}(x, y, z)+\left(\alpha_{0}+\alpha_{1} x\right) g(x, y, z)\right) e^{\alpha_{0} z} y^{\alpha_{1} / 2}
\end{aligned}
$$


Since

$$
\frac{d f}{d t}(x, y, z)=\left(\alpha_{0}+\alpha_{1} x\right) f(x, y, z)=\left(\alpha_{0}+\alpha_{1} x\right) g(x, y, z) e^{\alpha_{0} z} y^{\alpha_{1} / 2},
$$

from (4.7) and (4.8) we get that

$$
\frac{d g}{d t}(x, y, z) e^{\alpha_{0} z} y^{\alpha_{1} / 2}=0 \quad \text { that is } \quad \frac{d g}{d t}(x, y, z)=0 .
$$

In other words, either $g$ is a constant $\lambda \in \mathbb{C}$, or $g=g(x, y, z)$ is a first integral of system (1.1) which is a polynomial in the variable $x$. By Proposition 3.1 (with $\alpha=-1 / 2$ ) this last case is not possible. Hence from equation (4.6) we conclude that

$$
f(x, y, z)=\lambda e^{\alpha_{0} z} y^{\alpha_{1} / 2}
$$

Since $f$ is a polynomial we must have $\alpha_{0}=0$ and $\alpha_{1} / 2=m$ where $m$ is a non-negative integer. Furthermore $m \neq 0$, otherwise the cofactor of $f$ would be zero. Then

$$
f(x, y, z)=\lambda y^{m}, \quad \lambda \in \mathbb{C}, m \in \mathbb{N} \backslash\{0\},
$$

in contradiction with the fact that $f$ is an irreducible polynomial of degree greater or equal two. This completes the proof of the proposition.

Proposition 4.2. Assume that $\alpha \in \mathbb{C} \backslash\left\{\alpha_{n}: n=2,4, \ldots\right\}$ and $\alpha \neq-1 / 2$. Then, system (1.1) has no irreducible Darboux polynomial with non-zero cofactor.

Proof. It follows by direct computations that under the assumptions, system (1.1) has no Darboux polynomial of degree one. Now we shall prove that system (1.1) has no Darboux polynomials with non-zero cofactor of degree greater than or equal to two.

Assume that $f$ is an irreducible Darboux polynomial of system (1.1) with non-zero cofactor of degree $n \geq 2$. Then it satisfies

$$
\left(y-\frac{z}{2}-x^{2}\right) \frac{\partial f}{\partial x}+\left(\alpha+\frac{1}{2}+2 x y\right) \frac{\partial f}{\partial y}+\frac{\partial f}{\partial z}=\left(\alpha_{0}+\alpha_{1} x+\alpha_{2} y+\alpha_{3} z\right) f
$$

where $\left(\alpha_{0}, \alpha_{1}, \alpha_{2}, \alpha_{3}\right) \in \mathbb{C}^{4} \backslash\{(0,0,0,0)\}$. We separate the proof of this proposition into different parts.

First we prove that $\alpha_{2}=\alpha_{3}=0$. For this we assume that $\alpha_{2} \neq 0$ or $\alpha_{3} \neq 0$, and we write $f$ in sum of its homogeneous parts as $\sum_{j=0}^{n} f_{j}(x, y, z)$, where each $f_{j}$ is a homogeneous polynomial of degree $j$. Without loss of generality we can assume that $f_{n} \neq 0$. Computing the terms of degree $n+1$ we obtain (4.3), and the same arguments of the proof of Proposition 4.1 imply that $\alpha_{2}=\alpha_{3}=0$.

Now we have that equation (4.1) can be written in the form (4.5), where the derivative is evaluated along a solution of system (1.1). We write $f$ as in (4.6). Then $g$ is a polynomial in the variable 
$x$ and satisfies

$$
\begin{aligned}
\frac{d f}{d t}(x, y, z) & =\frac{d g}{d t}(x, y, z) e^{\alpha_{0} z} y^{\alpha_{1} / 2}+g(x, y, z)\left(\alpha_{0}+\alpha_{1} x+\frac{\alpha_{1}\left(\alpha+\frac{1}{2}\right)}{2 y}\right) e^{\alpha_{0} z} y^{\alpha_{1} / 2} \\
& =\left(\frac{d g}{d t}(x, y, z)+\left(\alpha_{0}+\alpha_{1} x+\frac{\alpha_{1}(\alpha+1 / 2)}{2 y}\right) g(x, y, z)\right) e^{\alpha_{0} z} y^{\alpha_{1} / 2}
\end{aligned}
$$

From (4.8) and (4.10) we get that

$$
\frac{d g}{d t}(x, y, z) e^{\alpha_{0} z} y^{\alpha_{1} / 2}=\frac{\alpha_{1}\left(\alpha+\frac{1}{2}\right)}{2 y} g(x, y, z) e^{\alpha_{0} z} y^{\alpha_{1} / 2},
$$

that is

$$
\frac{d g}{d t}(x, y, z)=\frac{\alpha_{1}(\alpha+1 / 2)}{2 y} g(x, y, z)
$$

and $g=g(x, y, z)$ is a polynomial in the variable $x$.

Now proceeding as in the proof of Proposition 3.1 (note that if we expand $g$ as powers of the variable $x$ as in (3.2) we have that $\sum_{j=0}^{n} \frac{\alpha_{1}\left(\alpha+\frac{1}{2}\right)}{2 y} g_{j}(y, z) x^{j}$ when compared with $\sum_{j=0}^{n} \frac{d g_{j}}{d z}(y, z) x^{j}$ will always belong to the 1.o.t. $(y)$, and hence it does not play any role because it does not interfere in the arguments used in the proof of Proposition 3.1). In short, we get that $g=g_{0}(y, z)$ and it satisfies

$$
\left(\alpha+\frac{1}{2}+2 x y\right) \frac{\partial g_{0}}{\partial y}+\frac{\partial g_{0}}{\partial z}=\frac{\alpha_{1}\left(\alpha+\frac{1}{2}\right)}{2 y} g_{0} .
$$

Since $g_{0}$ does not depend on $x$ we get that $\partial g_{0} / \partial y=0$ that is $g=g(z)$. Now we consider two cases.

Case 1: $\alpha_{1} \neq 0$. In this case since $g$ does not depend on $y$ we get that $\partial g / \partial z=0$ and then $g=0$. Therefore (4.6) imply that $f=0$, in contradiction with the fact that $f$ is a Darboux polynomial of degree greater than or equal to two.

Case 1: $\alpha_{1}=0$. In this case since $g$ does not depend on $y$ we get that $\partial g / \partial z=0$ and then $g=$ constant $=\lambda$. Therefore (4.6) imply that $f=\lambda e^{\alpha_{0} z}$. Since $f$ must be a polynomial we get $\alpha_{0}=0$ a contradiction with the fact that the cofactor $f$ is different from zero.

As usual $\mathbb{N}$ denotes the set of positive integers.

Proposition 4.3. Assume that $\alpha=-1 / 2$. Then the unique exponential factors of system (1.1) are $e^{z}, e^{z^{2} / 2}$ and $e^{2 y x^{2}-y^{2}+z y}$ with cofactors $1, z$ and $y$, respectively.

Proof. It follows from Proposition 2.1 that we can write

$$
E=e^{g / y^{n_{1}}} \quad \text { with } n_{1} \in \mathbb{N} \cup\{0\} \text { and }(g, y)=1,
$$

where $g$ is a polynomial satisfying

$$
\left(y-\frac{z}{2}-x^{2}\right) \frac{\partial g}{\partial x}+2 x y \frac{\partial g}{\partial y}+\frac{\partial g}{\partial z}-2 n_{1} x g=\left(\beta_{0}+\beta_{1} x+\beta_{2} y+\beta_{3} z\right) y^{n_{1}},
$$

where $\beta_{i} \in \mathbb{C}$ for $i=0,1,2,3$, and where we have simplified by the common factor $E$. We consider two cases 
Case 1: $n_{1} \in \mathbb{N}$. In this case restricting (4.11) to $y=0$ and denoting $\bar{g}=\left.g\right|_{y=0}$, we get

$$
-\left(\frac{z}{2}+x^{2}\right) \frac{\partial \bar{g}}{\partial x}+\frac{\partial \bar{g}}{\partial z}=2 n_{1} x \bar{g}
$$

Hence $\bar{g}$ is either zero or a Darboux polynomial of system (1.1) restricted to $y=0$. Since $g$ and $y$ are coprime, we have that $\bar{g} \neq 0$. We expand $\bar{g}$ in sum of its homogeneous parts as $\bar{g}=\sum_{j=0}^{n} \bar{g}_{j}(x, z)$, where each $\bar{g}_{j}$ is a homogeneous polynomial of degree $j$. Computing the terms of degree $n+1$ in (4.12) we get

$$
-x^{2} \frac{\partial \bar{g}_{n}}{\partial x}=2 n_{1} x \bar{g}_{n}
$$

Solving this partial differential equation we get that $\bar{g}_{n}=K(z) x^{-2 n_{1}}$ where $K$ is a function of $z$. Since $\bar{g}_{n}$ must be a polynomial we must have $K(z)=0$ and thus $\bar{g}_{n}=0$. Therefore $\bar{g}=0$ which is not possible.

Case 2: $n_{1}=0$. In this case $g$ satisfies

$$
\left(y-\frac{z}{2}-x^{2}\right) \frac{\partial g}{\partial x}+2 x y \frac{\partial g}{\partial y}+\frac{\partial g}{\partial z}=\beta_{0}+\beta_{1} x+\beta_{2} y+\beta_{3} z
$$

where $\beta_{i} \in \mathbb{C}$ for $i=0,1,2,3$. We define $G=E e^{-\beta_{0} z-\beta_{3} z^{2} / 2}$. Then using that $\frac{d E}{d t}=E \frac{d g}{d t}$ and (4.13), we get

$$
\begin{aligned}
\frac{d G}{d t} & =\frac{d E}{d t} e^{-\beta_{0} z-\beta_{3} z^{2} / 2}-\left(\beta_{0}+\beta_{3} z\right) E e^{-\beta_{0} z-\beta_{3} z^{2} / 2} \\
& =\left(\beta_{0}+\beta_{1} x+\beta_{2} y+\beta_{3} z\right) E e^{-\beta_{0} z-\beta_{3} z^{2} / 2}-\left(\beta_{0}+\beta_{3} z\right) E e^{-\beta_{0} z-\beta_{3} z^{2} / 2} \\
& =\left(\beta_{1} x+\beta_{2} y\right) G
\end{aligned}
$$

where the derivative is evaluated along a solution of system (1.1). Setting $G=e^{g-\beta_{0} z-\beta_{3} z^{2} / 2}=e^{h(x, y, z)}$ we have that

$$
\frac{d h}{d t}=\beta_{1} x+\beta_{2} y
$$

That is

$$
\left(y-\frac{z}{2}-x^{2}\right) \frac{\partial h}{\partial x}+2 x y \frac{\partial h}{\partial y}+\frac{\partial h}{\partial z}=\beta_{1} x+\beta_{2} y
$$

If we expand $h$ as $h=\sum_{j=0}^{n} h_{j}(y, z) x^{j}$ and proceeding as in the proof of Proposition 3.1 with $n \geq 4$ we get that $h_{n}=K(z) y^{n / 2}$ where $K(z)$ is a function in the variable $z$. Since $h_{n}$ must be a polynomial we conclude that $n$ must be even. Again proceeding as in the proof of Proposition 3.1 we get that $h_{n}=0$ (note that since $n \geq 4$ then $n-1 \geq 3$ and then when computing $h_{0}$ and $h_{1}$ we get that the 
left-hand side of equation (4.14) would be of the form

$$
(-1)^{n / 2+1} \gamma_{n} D_{n / 2} y^{n-1}+(-1)^{n / 2-1} E_{(n-2) / 2}(z) y^{n-1 / 2}+\text { 1.o.t }(y),
$$

while the right hand side of this equation will be in that case $\beta_{2} y$ which has degree one in the variable $y$. Since $n \geq 4$ even, this implies that $\gamma_{n}=0$ and then $h_{n}=0$ ). Hence we have that

$$
h(x, y, z)=h_{0}(y, z)+h_{1}(y, z) x+h_{2}(y, z) x^{2} .
$$

Furthermore we have that $h$ must satisfy

$$
\begin{aligned}
& \left(y-\frac{z}{2}-x^{2}\right)\left(2 h_{2}(y, z) x+h_{1}(y, z)\right)+2 x y\left(\frac{\partial h_{0}}{\partial y}+\frac{\partial h_{1}}{\partial y} x+\frac{\partial h_{2}}{\partial y} x^{2}\right) \\
& +\left(\frac{\partial h_{0}}{\partial z}+\frac{\partial h_{1}}{\partial z} x+\frac{\partial h_{2}}{\partial z} x^{2}\right)=\beta_{1} x+\beta_{2} y .
\end{aligned}
$$

Then computing the coefficient of $x^{3}$ in (4.15) we get

$$
-2 h_{2}(y, z)+2 y \frac{\partial h_{2}}{\partial y}(y, z)=0 \quad \text { that is } \quad h_{2}=K(z) y
$$

where $K(z)$ is a polynomial in the variable $z$. Now computing the coefficient of $x^{2}$ in (4.15) we obtain

$$
-h_{1}(y, z)+2 y \frac{\partial h_{1}}{\partial y}(y, z)+K^{\prime}(z) y=0, \quad \text { that is } \quad h_{1}=-K^{\prime}(z) y
$$

where we have used that $h_{1}$ must be a polynomial in the variables $y, z$.

Now computing the coefficient of $x$ in (4.15) we get

$$
\left(y-\frac{z}{2}\right) 2 K(z) y+2 y \frac{\partial h_{0}}{\partial y}-K^{\prime \prime}(z) y=\beta_{1} .
$$

Since the left-hand side of (4.16) is divisible by $y$ we get that $\beta_{1}=0$. Then after simplifying by $y$ we obtain that

$$
h_{0}=K_{1}(z)-\frac{K(z)}{2} y^{2}+\frac{K(z) z}{2} y+\frac{K^{\prime \prime}(z)}{2} y
$$

where $K_{1}(z)$ is a polynomial in the variable $z$.

Now computing the coefficient of $x^{0}$ in (4.15) we get

$$
-\frac{3}{2} K^{\prime}(z) y^{2}+\frac{K(z)}{2} y+K_{1}^{\prime}(z)+K^{\prime}(z) z y+\frac{K^{\prime \prime \prime}(z)}{2} y=\beta_{2} y .
$$

Computing the terms in (4.18) that do not depend on $y$ we obtain that $K_{1}^{\prime}(z)=0$. That is $K_{1}(z)$ is a constant that we take the constant zero, because it only affects the exponential $e^{h(x, y, z)}$ in a constant. 
Thus $K_{1}(z)=0$. Now after simplifying equation (4.18) by $y$ we get

$$
-\frac{3}{2} K^{\prime}(z) y+\frac{K(z)}{2}+K^{\prime}(z) z+\frac{K^{\prime \prime \prime}(z)}{2}=\beta_{2}
$$

The coefficient of $y$ in (4.19) yields $K^{\prime}(z)=0$. Therefore $K(z)=\gamma$ a constant. Hence equation (4.19) becomes

$$
-\frac{\gamma}{2}=\beta_{2} \quad \text { that is } \quad \gamma=-2 \beta_{2}
$$

This yields

$$
h=\beta_{2}\left(2 y x^{2}-y^{2}+z y\right) .
$$

So $g=h+\beta_{0} z+\beta_{3} z^{2} / 2=\beta_{2}\left(2 y x^{2}-y^{2}+z y\right)+\beta_{0} z+\beta_{3} z^{2} / 2$. This concludes the proof of the proposition.

Proposition 4.4. Assume $\alpha \in \mathbb{C} \backslash\left\{\alpha_{n}: n=2,4, \ldots\right\}$ and $\alpha \neq-1 / 2$. Then the unique exponential factors of system (1.1) are $e^{z}, e^{z^{2} / 2}$ and $e^{2 y x^{2}+(2 \alpha+1) x-y^{2}+z y}$ with cofactors $1, z$ and $y$, respectively.

Proof. Proceeding in an analogous way to the proof of Case 2 of Proposition 4.3, considering $E=$ $e^{g}, G=E e^{-\beta_{0} z-\beta_{3} z^{2} / 2}$ and $G=e^{h}$, we obtain that $h$ is of the form $h=h_{0}(y, z)+h_{1}(y, z) x+h_{2}(y, z) x^{2}$ and satisfies

$$
\begin{aligned}
& \left(y-\frac{z}{2}-x^{2}\right)\left(2 h_{2}(y, z) x+h_{1}(y, z)\right)+\left(\alpha+\frac{1}{2}+2 x y\right)\left(\frac{\partial h_{0}}{\partial y}+\frac{\partial h_{1}}{\partial y} x+\frac{\partial h_{2}}{\partial y} x^{2}\right) \\
& +\left(\frac{\partial h_{0}}{\partial z}+\frac{\partial h_{1}}{\partial z} x+\frac{\partial h_{2}}{\partial z} x^{2}\right)=\beta_{1} x+\beta_{2} y .
\end{aligned}
$$

Computing the coefficient of $x^{3}$ in (4.20) and proceeding as in the proof of Case 2 of Proposition 4.3 we get $h_{2}=K(z) y$, where $K(z)$ is a polynomial in the variable $z$. Now computing the coefficient of $x^{2}$ in (4.20) we obtain

$$
-h_{1}(y, z)+2 y \frac{\partial h_{1}}{\partial y}(y, z)+\left(\alpha+\frac{1}{2}\right) K(z)+K^{\prime}(z) y=0
$$

that is $h_{1}=\left(\alpha+\frac{1}{2}\right) K(z)-K^{\prime}(z) y$, where we have used that $h_{1}$ must be a polynomial in the variables $y, z$.

Now computing the coefficient of $x$ in (4.20) we get

$$
\left(y-\frac{z}{2}\right) 2 K(z) y+2 y \frac{\partial h_{0}}{\partial y}-K^{\prime \prime}(z) y=\beta_{1}
$$

which is equation (4.16). Thus $\beta_{1}=0$ and $h_{0}$ is of the form (4.17). 
Now computing the coefficient of $x^{0}$ in (4.20) we get

$$
-\frac{3}{2} K^{\prime}(z) y^{2}+\frac{K(z)}{2} y+K_{1}^{\prime}(z)+K^{\prime}(z) z y+\frac{K^{\prime \prime \prime}(z)}{2} y+\left(\alpha+\frac{1}{2}\right) \frac{K^{\prime \prime}(z)}{2}=\beta_{2} y .
$$

Computing the coefficient of $y^{2}$ in (4.22) we get $K^{\prime}(z)=0$. Thus $K(z)=\gamma \in \mathbb{C}$ and (4.22) becomes

$$
\frac{\gamma}{2} y+K_{1}^{\prime}(z)=\beta_{2} y, \quad \text { that is } \quad \gamma=2 \beta_{2} \quad \text { and } \quad K_{1}^{\prime}(z)=0 .
$$

Again we take $K_{1}(z)=0$. This yields

$$
h=\beta_{2}\left(2 y x^{2}+(2 \alpha+1) x-y^{2}+z y\right) .
$$

So $g=h+\beta_{0} z+\beta_{3} z^{2} / 2=\beta_{2}\left(2 y x^{2}+(2 \alpha+1) x-y^{2}+z y\right)+\beta_{0} z+\beta_{3} z^{2} / 2$. This concludes the proof of the proposition.

Proof of Theorem 1.3. Statement (c.1) follows from Corollary 4.1. Statement (a) follows from Proposition 4.1. Proposition 4.2 shows statement (b). Finally, statement (c.2) follows from Propositions 4.3 and 4.4 .

\section{Proof of Theorem 1.2}

The proof of Theorem 1.2 will be done by contradiction. Assume that $G$ is a first integral of system (1.1) of Darboux type. We distinguish between two cases.

Case 1: $\alpha=-1 / 2$. From the definition of Darboux first integral in (2.4), and taking into account Theorem $1.3 \mathrm{G}$ must be of the form

$$
G=y^{\lambda_{1}} e^{\mu_{1} z} e^{\mu_{2} z^{2} / 2} e^{\mu_{3}\left(2 y x^{2}-y^{2}+y z\right)}, \quad \lambda_{1}, \mu_{1}, \mu_{2}, \mu_{3} \in \mathbb{C} .
$$

Since $G$ is a first integral of system (1.1) it must satisfy

$$
0=\left(y-\frac{z}{2}-x^{2}\right) \frac{\partial G}{\partial x}+2 x y \frac{\partial G}{\partial y}+\frac{\partial G}{\partial z}=\left(2 \lambda_{1} x+\mu_{1}+\mu_{2} z+\mu_{3} y\right) G .
$$

Hence

$$
2 \lambda_{1} x+\mu_{1}+\mu_{2} z+\mu_{3} y=0, \quad \text { that is } \quad \lambda_{1}=\mu_{1}=\mu_{2}=\mu_{3}=0 .
$$

Therefore $G$ is a constant in contradiction with the fact that $G$ is a first integral.

Case 2: $\alpha \in \mathbb{C} \backslash\left\{\alpha_{n}: n=2,4, \ldots\right\}$ and $\alpha \neq-1 / 2$. From the definition of Darboux first integral and taking into account Theorem $1.3 \mathrm{G}$ must be of the form

$$
G=e^{\mu_{1} z} e^{\mu_{2} z^{2} / 2} e^{\mu_{3}\left(2 y x^{2}+(2 \alpha+1) x-y^{2}+y z\right)}, \quad \mu_{1}, \mu_{2}, \mu_{3} \in \mathbb{C} .
$$

Since $G$ is a first integral of system (1.1) it must satisfy

$$
0=\left(y-\frac{z}{2}-x^{2}\right) \frac{\partial G}{\partial x}+\left(\alpha+\frac{1}{2}+2 x y\right) \frac{\partial G}{\partial y}+\frac{\partial G}{\partial z}=\left(\mu_{1}+\mu_{2} z+\mu_{3} y\right) G
$$

Hence

$$
\mu_{1}+\mu_{2} z+\mu_{3} y=0, \quad \text { that is } \mu_{1}=\mu_{2}=\mu_{3}=0 .
$$

Therefore $G$ is a constant in contradiction with the fact that $G$ is a first integral. This completes the proof of Theorem 1.2. 


\section{Acknowledgements}

The first author is partially supported by a MICINN/FEDER grants numbers MTM2009-03437 and MTM2013-40998-P, by an AGAUR grant number 2014SGR-568, by an ICREA Academia, two FP7+PEOPLE+2012+IRSES numbers 316338 and 318999, and FEDER-UNAB10-4E-378. The second author is supported by Portuguese National Funds through FCT - Fundação para a Ciência e a Tecnologia within the projects PTDC/MAT/117106/2010 and PEst-OE/EEI/LA0009/2013 (CAMGSD).

\section{References}

[1] C. Christopher, J. Llibre and J.V. Pereira, Multiplicity of invaraiant algebraic curves and Darboux integrability, Pacific J. Math. 229 (2007), 63-117.

[2] J. Llibre and C. Valls, Darboux integrability of a simplified Friedman-Robertson-Walker Hamiltonian system, J. Nonlinear Mathematical and Physics 19 2012, 1250036-pp 11.

[3] J. Malmquist, Sur les équations différentielles du second ordre dont l'intégrale génerale a ses points critiques fixes, Arkiv. Mat. Astron. Fys. 18 (1922), 1-89.

[4] J.J. Morales, A remark about the Painlevé transcendents. Théories asymptotiques et équations de Painlevé, Sémin. Congr. 14 (2006), Soc. Math. France, Paris, 229-235.

[5] K. Okamoto, Polynomial Hamiltonians associated to Painlevé equations I, II, Proc. Japan Acad. Ser. A 56 (1980), 264-268; 367-371. 